\title{
Mechanisms of the sperm guidance, an essential aid for meeting the oocyte
}

\author{
Raquel Lottero-Leconte*, Carlos Agustín Isidro Alonso*, Luciana Castellano, Silvina Perez Martinez \\ Laboratory of Biology of Reproduction in Mammals, Center for Pharmacological and Botanical Studies (CEFYBO-CONICET), School of \\ Medicine, University of Buenos Aires (UBA), Buenos Aires, Argentina \\ *These authors contributed equally to this work. \\ Correspondence to: Silvina Perez Martinez, Senior Investigator. Center for Pharmacological and Botanical Studies, University of Buenos Aires (UBA), \\ School of Medicine, National Scientific and Technical Research Council-Argentina (CONICET), Paraguay 2155, 15th Floor, C1121ABG, Ciudad \\ de Buenos Aires, Argentina. Email: perezms@fmed.uba.ar. \\ Provenance: This is an invited Editorial commissioned by Section Editor Weijun Jiang (Nanjing Normal University, Department of Reproductive and \\ Genetics, Institute of Laboratory Medicine, Jinling Hospital, Nanjing University School of Medicine, Nanjing, China). \\ Comment on: De Toni L, Garolla A, Menegazzo M, et al. Heat Sensing Receptor TRPV1 Is a Mediator of Thermotaxis in Human Spermatozoa. \\ PLoS One 2016;11:e0167622.
}

Submitted Mar 07, 2017. Accepted for publication Mar 14, 2017.

doi: $10.21037 /$ tcr.2017.03.68

View this article at: http://dx.doi.org/10.21037/tcr.2017.03.68

In mammals, ejaculated spermatozoa must migrate into the female reproductive tract in order to reach and fertilize the oocyte (Figure 1). The number of spermatozoa that reach the oviductal isthmus (where they attach to oviductal cells and form the sperm reservoir) is small $(1,2)$ and only $~ 10 \%$ of these spermatozoa in humans become capacitated (3) and acquire a state of readiness for fertilizing the oocyte. In addition, the sperm cells have a torturous and long way between the reservoir and the oocyte at the fertilization site (3-5 cm in humans) (2). These facts, together with the tiny dimensions of the gametes in comparison to the tube length make improbable the sperm arrival to the fertilization site and make evident the need for sperm guidance (4). Different guidance mechanisms appear essential for successful sperm arrival to the fertilization site: the short range mechanism such as chemotaxis (swimming up a chemoattractant gradient) (5) and the long range mechanisms such as rheotaxis (swimming against a fluid flow) and thermotaxis (the temperature-oriented cell motility) (Figure 1).

When spermatozoa become capacitated, they acquire a number of properties that render them fertilizing ability. Thermotaxis is one of these properties in which sperm cells acquire the ability to be thermotactically active (6). This property is manifested by the ability of the capacitated spermatozoa to change their swimming direction according to a temperature gradient (towards the warmer temperature) (Figure 1). Spermatozoa can sense both the absolute ambient temperature and the temperature gradient. Previous studies showed that, at peri-ovulation stage, there is a temperature difference between the sperm reservoir site (cooler) and the fertilization site (warmer). Thus, a temperature difference of $\sim 2{ }^{\circ} \mathrm{C}$ exists in rabbits between the isthmus and the isthmicampullary junction (7) and $\sim 0.7^{\circ} \mathrm{C}$ difference in mated pigs between the isthmus and the ampulla (8). Subsequently, Bahat and collaborators (9) demonstrated that rabbit and human spermatozoa can sense small temperature differences and respond to it by thermotaxis. These findings raised the hypothesis that the temperature difference might serve as a cue for guiding spermatozoa to the site of fertilization. Interestingly, Bahat et al., (10) recently has reported that human spermatozoa are thermotactically responsive over a wide temperature range $\left(29-41^{\circ} \mathrm{C}\right)$, much wider than the range considered physiological.

As in sperm chemotaxis, only capacitated spermatozoa are thermotactically responsive (9). Additionally, in chemotaxis and thermotaxis, spermatozoa swim up the gradient by modulating the frequency of turns and hyperactivation events, essentially in the direction of the gradient $(11,12)$.

The molecular bases of sperm thermotaxis remain poorly understood. Taking into account that thermotaxis favours 


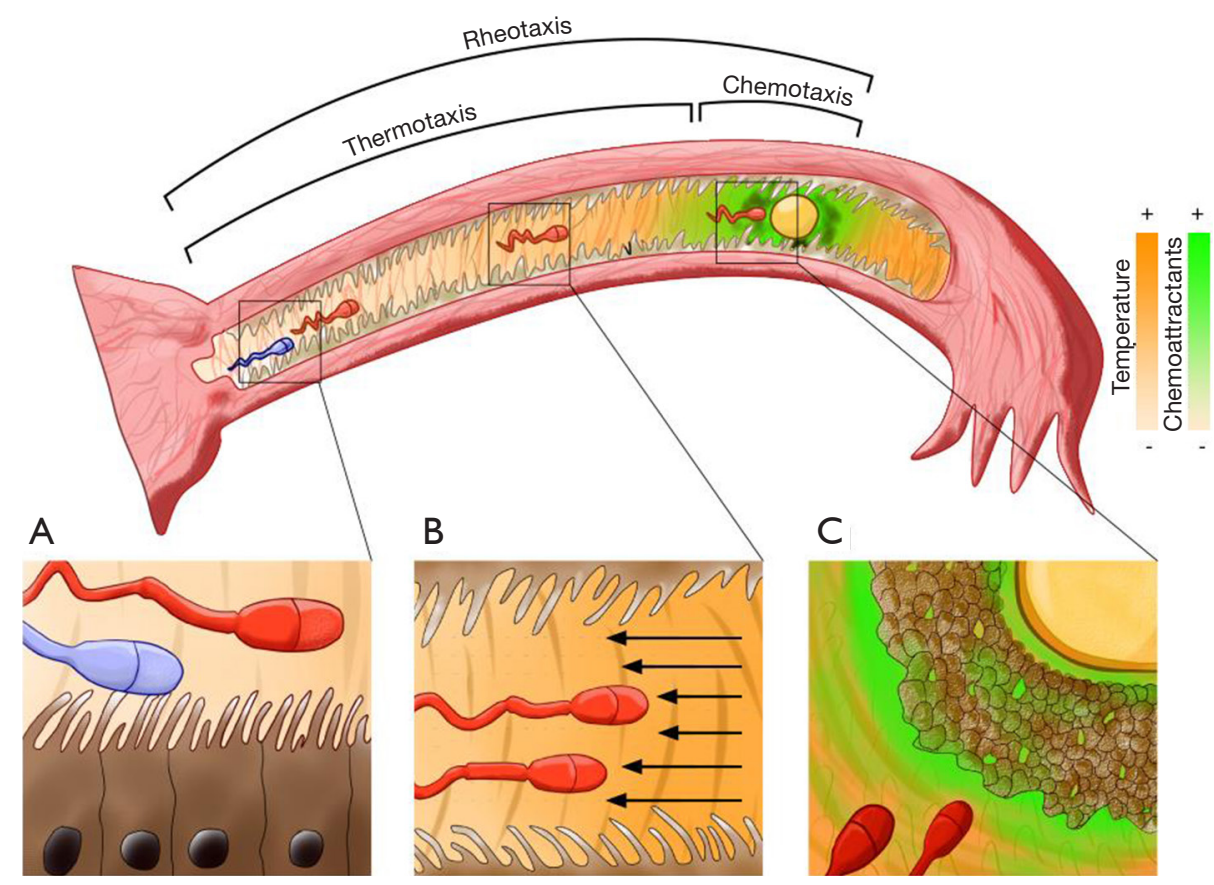

Figure 1 Schematic representation of the sperm travel through the oviduct. Guidance of spermatozoa to the oocyte is mainly conducted by two mechanisms: (I) long range mechanisms such as rheotaxis or thermotaxis, and (II) short range mechanisms like chemotaxis. (A) Once spermatozoa become capacitated in the lower region of the oviduct (isthmus) they are rendered a hyperactivated movement of the flagellum, a higher sensitivity to temperature gradient, and a partial loose of the ability to bind oviductal epithelial cell; (B) hyperactivated movement is essential to counteract the flow of the oviduct that, in time, helps spermatozoa to redirect their way and force them to swim straight; (C) in the upper region of the oviduct (ampulla), certain chemical compounds produced by the cumulus and the oocyte itself guide spermatozoa towards their final destination. (Illustration by Carlos Agustín Alonso, 2017).

the sperm-egg communication and therefore fertilization, and that these events require intracellular calcium $\left(\mathrm{Ca}^{+}\right)$ changes (13), most works regarding molecular mechanisms involved in thermotaxis are focused on molecules modulating of $\mathrm{Ca}^{+}$levels. In this sense, Bahat and collaborators (14), reported that the activation of the molecular pathway of phospholipase C/IP3R, but not the transient receptor potential (TRP) channels, is involved in human sperm thermotaxis. However, Mondal and collaborators (15) showed the involvement of the $\mathrm{Ca}^{+}$channel TRPV4 in mouse sperm thermotaxis. This evidence was confirmed by the work of Hamano and collaborators (16) that examined thermotaxis in TRPV $4^{-/-}$mouse sperm.

On the other hand, Martínez-López (17) found that mouse sperm possess TRPM8 channel, which is sensible to temperature changes and might be involved in thermotaxis. In addition, TRPM8 may influence the acrosome reaction.

The work of De Toni and collaborators (18), is focused on the study of TRPV1 as a molecular modulator of thermotaxis in human spermatozoa. Transient Receptor Potential Cation Channel or Vanilloid Receptor 1 (TRPV1) is a ligand-gated, nonselective cationic channel that is activated by molecules derived from plants, such as capsaicin, and also by stimuli like heat or protons (19). It has been reported that anandamide (AEA), an endocannabinoid synthesized by sperm and oviductal cells, activates TRPV1 and the ligand-receptor interaction occurs through a cytosolic binding site triggering an increase of intracellular $\mathrm{Ca}^{+}$concentration and/or activation of protein kinases (20). TRPV1 is expressed in mammalian spermatozoa and its activation is involved in the regulation of sperm oviduct interaction, sperm capacitation and acrosome reaction (21-23). On the other hand, sperm release from the oviductal reservoir depends on $\mathrm{Ca}^{+}$influx by activation of cannabinoid type 1 receptor and TRPV1 by AEA (22).

The study of De Toni shows the involvement of TRPV1 in thermotaxis through the evaluation of the sperm 
migration toward a temperature gradient that was reduced by the addition of selective antagonists of TRPV1.

Several works indicated that only capacitated spermatozoa can undergo thermotaxis; however, in De Toni's work both capacitated and non-capacitated spermatozoa responded to thermotactic stimulus. Capacitated spermatozoa showed higher response, thus the authors suggest that capacitation essentially amplifies the cell response to the temperature gradient. This is also supported by the fact that only the spermatozoa that enter the oviduct can undergo sperm capacitation and might be able to sense the thermotactic stimulus that is the difference existing between the storage and the fertilization site.

On the other hand, this work demonstrates sperm migration toward a gradient of capsaicin, an active component of chili peppers and a selective agonist of TRPV1, in a concentration dependent manner. This suggests that TRPV1 might be involved in the chemotaxis mechanism. Interestingly, cell migration by thermotaxis or capsaicin gradient is able to select spermatozoa with higher expression of TRPV1. In this work, a possible synergistic or additive effect of capsaicin in the presence of a temperature gradient is not shown. This aspect might be interesting to consider for future works.

Recently, it has been demonstrated that the bovine oviductal fluid contains nanomolar concentrations of AEA which fluctuate during the estrous cycle, with highest levels detected during the peri-ovulatory period (24). In addition, several works support the evidence that spermatozoa are normally exposed to this lipid mediator within the reproductive tract (20). Therefore, the presence of this endocannabinoid in follicular fluids at ovulation, suggests that the mature follicle may contribute to oviductal AEA levels to create an endocannabinoid gradient conducive to the regulation of sperm function. In this sense, AEA (as natural agonist), might mimic the migration effects of capsaicin involving TRPV1 activation, supporting the observations reported by De Toni et al.

De Toni's work also showed that TRPV1 antagonists decreased intracellular $\mathrm{Ca}^{+}$levels of cells migrated toward either temperature or capsaicin gradients. These data support $\mathrm{Ca}^{+}$-flux properties of TRPV1 receptor/channel and the increase of $\mathrm{Ca}^{+}$-dependent hyperactivation (13) observed during sperm thermotaxis.

It has to be taken into account that the number of stimuli inside the oviduct as well as the responsive mechanisms to gradients, might be greater than the ones studied in this work, and therefore, a partial reversion with the antagonists used is of great value to the understanding of this process.

The study of the molecular bases of thermotaxis could contribute not only to the knowledge of this physiological process but also to the development of advanced therapeutic strategies for treating male infertility. In this sense, recently it has been reported that different thermotaxis response between bull sperm samples may be associated with sperm fertility (15).

In addition, the role of TRPV1 as a mediator of sperm thermotaxis in humans may represent a novel molecular target for the selection of sperm cells with fertilizing ability. Altogether, thermotaxis may be a reliable tool for analyzing sperm migration and has potential as a predictor of sperm quality, and therefore sperm fertilizing ability.

\section{Acknowledgements}

The authors thank Carlos Agustin Alonso for his help in Figure 1 design and drawing.

Funding: This manuscript was supported by grant from Agencia Nacional de Promoción Científica y Tecnológica (PICT 2014 2325).

\section{Footnote}

Conflicts of Interest: The authors have no conflicts of interest to declare.

\section{References}

1. Suarez SS. Formation of a reservoir of sperm in the oviduct. Reprod Domest Anim 2002;37:140-3.

2. Sun F, Bahat A, Gakamsky A, et al. Human sperm chemotaxis: both the oocyte and its surrounding cumulus cells secrete sperm chemoattractants. Hum Reprod 2005;20:761-7.

3. Cohen-Dayag A, Tur-Kaspa I, Dor J, et al. Sperm capacitation in humans is transient and correlates with chemotactic responsiveness to follicular factors. Proc Natl Acad Sci U S A 1995;92:11039-43.

4. Eisenbach M. Tur-Kaspa I. Do human eggs attract spermatozoa? Bioessays 1999;21:203-10.

5. Perez-Cerezales S, Boryshpolets S, Eisenbach M. Behavioral mechanisms of mammalian sperm guidance. Asian J Androl 2015;17:628-32.

6. Eisenbach M, Giojalas LC. Sperm guidance in mammals - an unpaved road to the egg. Nat Rev Mol Cell Biol 2006;7:276-85. 
7. David A, Vilensky A, Nathan H. Temperature changes in different parts of the rabbit oviduct. Preliminary report. Harefuah 1971;80:180-2.

8. Hunter RH, Nichol R. A preovulatory temperature gradient between the isthmus and ampulla of pig oviducts during the phase of sperm storage. J Reprod Fertil 1986;77:599-606.

9. Bahat A, Tur-Kaspa I, Gakamsky A, et al. Thermotaxis of mammalian sperm cells: a potential navigation mechanism in the female genital tract. Nat Med 2003;9:149-50.

10. Bahat A, Caplan SR, Eisenbach M. Thermotaxis of human sperm cells in extraordinarily shallow temperature gradients over a wide range. PLoS One. 2012;7:e41915.

11. Armon L, Eisenbach M. Behavioral mechanism during human sperm chemotaxis: involvement of hyperactivation. PLoS One 2011;6:e28359.

12. Boryshpolets S, Pérez-Cerezales S, Eisenbach M. Behavioral mechanism of human sperm in thermotaxis: a role for hyperactivation. Hum Reprod 2015;30:884-92.

13. Darszon A, Nishigaki T, Beltran C, et al. Calcium channels in the development, maturation, and function of spermatozoa. Physiol Rev 2011;91:1305-55.

14. Bahat A, Eisenbach M. Human sperm thermotaxis is mediated by phospholipase $\mathrm{C}$ and inositol trisphosphate receptor Ca2 + channel. Biol Reprod 2010;82:606-16.

15. Mondal MA, Takagi Y, Baba SA, et al. Involvement of calcium channels and intracellular calcium in bull sperm thermotaxis. J Reprod Dev 2016. [Epub ahead of print].

16. Hamano K, Kawanishi T, Mizuno A, et al. Involvement of

Cite this article as: Lottero-Leconte R, Alonso CA, Castellano L, Perez Martinez S. Mechanisms of the sperm guidance, an essential aid for meeting the oocyte. Transl Cancer Res 2017;6(Suppl 2):S427-S430. doi: 10.21037/tcr.2017.03.68
Transient Receptor Potential Vanilloid (TRPV) 4 in mouse sperm thermotaxis. J Reprod Dev 2016;62:415-22.

17. Martínez-López P, Treviño CL, de la Vega-Beltrán JL, et al. TRPM8 in mouse sperm detects temperature changes and may influence the acrosome reaction. J Cell Physiol 2011;226:1620-31.

18. De Toni L, Garolla A, Menegazzo M, et al. Heat Sensing Receptor TRPV1 Is a Mediator of Thermotaxis in Human Spermatozoa. PLoS One 2016;11:e0167622.

19. Ross RA. Anandamide and vanilloid TRPV1 receptors. $\mathrm{Br}$ J Pharmacol 2003;140:790-801.

20. Wang H, Dey SK, Maccarrone M. Jekyll and hyde: two faces of cannabinoid signaling in male and female fertility. Endocr Rev 2006;27:427-48.

21. Gervasi MG, Osycka-Salut C, Caballero J, et al. Anandamide capacitates bull spermatozoa through CB1 and TRPV1 activation. PLoS One 2011;6:e16993.

22. Gervasi MG, Osycka-Salut C, Sanchez T, et al. Sperm Release From the Oviductal Epithelium Depends on $\mathrm{Ca}(2+)$ Influx Upon Activation of CB1 and TRPV1 by Anandamide. J Cell Biochem 2016;117:320-33.

23. Francavilla F, Battista N, Barbonetti A, et al. Characterization of the endocannabinoid system in human spermatozoa and involvement of transient receptor potential vanilloid 1 receptor in their fertilizing ability. Endocrinology 2009;150:4692-700.

24. Gervasi MG, Marczylo TH, Lam PM, et al. Anandamide levels fluctuate in the bovine oviduct during the oestrous cycle. PLoS One 2013;8:e72521. 